J. Lake Sci. (湖泊科学), $2006, \mathbf{1 8}(5): 528-534$

http:// www. jlakes. org. E-mail: jlakes@ niglas. ac.cn

(c) 2006 by Journal of Lake Sciences

\title{
太湖水体中悬浮物的静沉降特征
}

\author{
陈 釷 ${ }^{1,2}$, 高 光 ${ }^{1 * *}$, 李一平 ${ }^{3}$, 王 珂 ${ }^{3}$, 逢 勇 $^{3}$ \\ ( $1:$ 中国科学院南京地理与湖泊研究所,南京 210008) \\ (2: 中国科学院研究生院, 北京 100039) \\ ( 3 : 河海大学环境科学与工程学院,南京 210098)
}

\begin{abstract}
摘 要: 本文分别采用斯托克斯沉降速率公式和重复深度吸管法计算了 2005 年 4 月、5 月间在太湖进行的四次静沉降模 拟实验中的沉降速度. 结果表明: 1) 太湖水体中悬浮物的沉降属于絮凝沉降. 2) 水体中悬浮物浓度与沉降时间均呈现出 明显的指数衰减规律 $\left(R^{2}>0.80\right)$, 悬浮物中无机物含量较高时这种规律更为明显 $\left.\left(R^{2} \geqslant 0.99\right) .3\right)$ 悬浮物浓较低时, 太湖 悬浮物的沉降速率与水体中的悬浮物浓度无明显的相关关系; 而悬浮物浓度较高时, 沉降速率随悬浮物浓度升高而增大. 经拟合沉降速度 $(\omega)$ 与悬浮物浓度 $(C)$ 之间符合 Logistic 曲线 $\omega=0.021 /(1+\exp (-0.026(C-166.3))), R^{2}=0.98$, $n=54.4)$, 斯托克斯公式可用来粗略估算太湖悬浮物的沉降速率, 而重复深度吸管法则适合于较精确地计算太湖悬浮物 的沉降速率, 但在计算时须注意根据悬浮物的特性, 选取其特征沉降速率. 本文计算得到的太湖悬浮物的沉降速率范围 为 $0.002 \mathrm{~cm} / \mathrm{s}-0.005 \mathrm{~cm} / \mathrm{s}$.
\end{abstract}

关键词: 悬浮物; 静沉降; 沉降速度; 太湖

\section{Hydrostatic settling of suspended matter of Lake Taihu}

CHEN Jun ${ }^{1,2}$, GAO Guang ${ }^{1}$, LI Yiping ${ }^{3}$, WANG Ke ${ }^{3}$ \& PANG Yong ${ }^{3}$

(1: Nanjing Institute of Geography and Limnology, Chinese Academy of Sciences, Nanjing 210008, P. R. China)

(2: Graduate School of Chinese Academy of Sciences, Beijing 100039, P. R. China)

(3: College of Environment Science and Engineering, Hohai University, Nanjing, 210098, P. R. China)

Abstract: Four experiments were conducted in laboratory for hydrostatic settling behavior of suspended matter in Lake Taihu in April and May, 2005. The settling velocity of suspended matter was calculated by both Stokes equation and McLaughlin method, and the results were further compared. The results showed that settlement of suspended matter in Lake Taihu was flocculation settlement. It also showed that the suspended matter concentration decayed exponentially with time, which was more obvious when the percentage of inorganic suspended matter accounting for total suspended matter was higher $\left(R^{2} \geqslant 0.99\right)$. When the suspended matter concentration was low, no clear relationship was found between settling velocity and suspended matter concentration. But settling velocity obviously rose with the increase of suspended matter concentration while the latter was high. Based on the data of four hydrostatic experiments, it was found that settling velocity $(\omega)$ of suspended matter and suspended matter concentration ( $C$ ) fitted in Logistic Curve $\omega=0.021 /(1+\exp (-0.026(C-166.3))), R^{2}=0.98, n=54$. Comparing two calculation methods, Stokes equation could be used to estimate the settling velocity, but the calculation result was more accurate using Mclaughlin method. The settling velocity of suspended matter estimated in Lake Taihu ranged from $0.002 \mathrm{~cm} / \mathrm{s}$ to $0.005 \mathrm{~cm} / \mathrm{s}$.

Keywords : suspended matter; hydrostatic settling; settling velocity; Lake Taihu

* 国家自然科学基金项目 (40573062)、国家重点基础研究发展计划项目 (2002CB412305)联合资助. $2005-11-05$ 收稿, 2005-12-20 收修改稿. 陈釙, 女, 1983 年生, 研究生.

** 通信联系人,guanggao@ niglas. ac.cn. 
对于大型浅水湖泊而言, 其沉积物在风浪的作用下极易发生再悬浮 ${ }^{[1,2]}$. 水体中的这些悬浮物在沉降 过程中, 通过吸附、絮凝等作用往往与水体中的营养盐、其他颗粒及微生物结合在一起. 因此, 水体中悬浮 物的沉降对水生生态系统中有机物的迁移、转化以及与其相伴随的营养盐循环再生具有重要意义 ${ }^{[3,4]}$. 此 外悬浮物的沉降速率也是生态系统热力学 ${ }^{[5]}$ 和动力学模型 ${ }^{[6]}$ 的重要输人参数. 一般而言, 水体中悬浮物的 沉降方式有两种. 一种是粒径较大的颗粒, 通常是以单颗粒的方式进行沉降, 即通常所谓的泥沙沉降; 另一 种是水体中粒径 $\mathrm{d}<0.03 \mathrm{~mm}$ 的细颗粒悬浮物, 虽然这类悬浮物在总悬浮物中所占的比例不大, 但由于其富 含有机物质, 且其在沉降时往往与水体中的其它颗粒结合在一起, 通常以絮凝的方式进行沉降, 从而使得其 实际沉降速率远大于相同粒径单颗粒泥沙的沉降速率 ${ }^{[7,8]}$. 事实上,水体中悬浮颗粒物的沉降速率除与粒 径密切相关外,还受多种环境因素的影响,有关这方面的研究近年来日益受到国际上的重视 ${ }^{[9-13]}$. 研究表 明,悬浮物浓度是影响悬浮颗粒物沉降速率的最重要因素,而悬浮物组成、含量以及水环境化学特征的不同 亦可通过物理、化学、生物作用影响颗粒物的絮凝能力,进而影响其沉降速率. 已有的研究成果主要集中于 海洋、港口、河口等, 而浅水湖泊则少见报道. 位于经济发达的长江下游三角洲地区的太湖,污染程度较为 严重,有机质含量较高, 其沉降机理及特性可能有别于海洋、港口等,但该方面的研究尚处于初期. 为初步 了解太湖悬浮物的沉降性能及沉降速度,2005 年 4 月、5 月间在太湖进行了几次悬浮物的静沉降实验. 通 过在不同风浪条件下,采集具有不同悬浮物浓度的太湖原水,模拟了太湖悬浮物在不同初始浓度下的沉降 过程, 分别采用斯托克斯沉降速率公式和重复深度吸管法计算了太湖悬浮物的沉降速率, 并对这两种方法 计算的结果进行了比较, 以期了解太湖水体中悬浮物在静止状态下,沉降过程中随时间的变化特征,便于进 一步研究实际运动状态下的沉积速率, 为进一步揭示太湖沉积物悬浮沉降规律、悬浮物沉降对水下光场、初 级生产力的影响和内源释放规律提供基础支撑.

\section{1 材料与方法}

\section{1 实验装置}

实验装置为有机玻璃圆筒, 内径 $19 \mathrm{~cm}$,外径 $20 \mathrm{~cm}$, 截面积 $284 \mathrm{~cm}^{2}$, 高 $54 \mathrm{~cm}$. 在筒壁上从上至下设置 3 个取样口, 上层取样口距离沉降筒顶端 $14 \mathrm{~cm}$, 上中层高度差 $20 \mathrm{~cm}$, 中下层高度差 $16 \mathrm{~cm}$, 下层取样口距离筒 底 $4 \mathrm{~cm}$.

\section{2 取样时间和方法}

室内静沉降实验共进行 4 次, 实验时间分别为 2005 年 4 月 $7-8$ 日, 2005 年 4 月 8-9 日, 2005 年 5 月 $28-29$ 日, 2005 年 5 月 30-31 日. 实验用水均取自中国科学院太湖湖泊生态系统研究站 (简称太湖站) 栈 桥附近的太湖原水, 取样后立即送人实验室中进行实验. 在将原水注人沉降筒之前, 再次摇匀, 然后注满沉 降筒, 同时取初始水样 $50 \mathrm{ml}$. 实验过程中, 分上、中、下 3 层同时取样, 取样体积均为 $50 \mathrm{ml}$. 取样时间间隔 为 $0 、 5 、 10 、 15 、 20 、 30 、 40 、 60 、 90 、 120 、 150 、 180 、 240 、 300 、 480 、 720 、 1440 、 2160 \mathrm{~min}$. 实验期间, 水温保持在 $25^{\circ} \mathrm{C}$ 左右.

\section{3 测定项目及分析方法}

悬浮物及有机颗粒物的测定 ${ }^{[2]}$ : 用已经 $105^{\circ} \mathrm{C}$ 烘干并称重 $\left(W_{1}\right)$ 的 Whatman $\mathrm{GF} / \mathrm{C}$ 滤膜抽滤水样. 抽滤 后, 滤膜恒温 $105^{\circ} \mathrm{C}$ 烘 $4 \mathrm{~h}$, 冷却平衡后称重 $\left(W_{2}\right)$, 继而放人马弗炉 $550^{\circ} \mathrm{C}$ 灼烧 $5 \mathrm{~h}$, 冷却平衡后称重 $\left(W_{3}\right)$. $W_{2}-W_{1}$ 为总悬浮物重, $W_{2}-W_{3}$ 即为有机颗粒物重. 颗粒物粒度用 Malven Masterizer 2000 激光粒度仪测定, 仪 器的测量范围为:0.02-2000 $\mu \mathrm{m}$, 重复测量误差小于 $1 \%$.

\section{4 沉降速率计算方法}

本文采用两种方法进行计算:斯托克斯沉降速率公式和重复深度吸管法.

1.4.1 斯托克斯沉降速率公式 由于 4 次实验中,悬浮物的中值粒径均在 $0.007-0.011 \mathrm{~mm}$ 之间 (表 1), 属 于粘性细颗粒泥沙范畴, 对于粘性细颗粒泥沙 $\left(d<0.1 \mathrm{~mm}, R_{e}<0.5\right)$, 斯托克斯公式应修订为如下形式 ${ }^{1}$ :

(1) 张金善, 陆培东. 太湖泥沙静水沉降特征的初步实验研究. 南京水利科学研究院报告, 2002 . 


$$
\omega=\frac{g}{24 v}\left(\frac{\gamma_{s}-\gamma}{\gamma}\right) d^{2}
$$

式中, $g$ 为重力加速度 $\left(\mathrm{m} / \mathrm{s}^{2}\right), v$ 为水的运动学粘性系数 $\left(\mathrm{m}^{2} / \mathrm{s}\right), \gamma_{s}$ 和 $\gamma$ 分别为泥沙和水的容重 $\left(\mathrm{N} / \mathrm{m}^{3}\right), d$ 和 $\omega$ 分别为泥沙的粒径 $(\mathrm{mm})$ 及沉降速率 $(\mathrm{cm} / \mathrm{s})$.

1.4.2 重复深度吸管法 由于县浮泥沙在静水中絮凝沉降基本上是一维的. 1961 年 T. McLaughlin 从输沙 平衡方程式出发, 推导出在静水条件下有 ${ }^{[14]}$ :

$$
\frac{\partial S}{\partial t}+\frac{\partial(\bar{\omega} S)}{\partial z}=0
$$

式中, $S$ 为含沙浓度 $(\mathrm{mg} / \mathrm{L}), t$ 为时间 $(\mathrm{s}), \bar{\omega}$ 为泥沙平均沉降速率 $(\mathrm{cm} / \mathrm{s}), z$ 为水深 $(\mathrm{cm})$.

对上式中的 $z$ 积分得:

$$
(\bar{\omega} S)_{z=H}=-\int_{0}^{H} \frac{\partial S}{\partial t} \mathrm{~d} z=-\frac{\partial}{\partial t} \int_{0}^{H} s \mathrm{~d} z
$$

通过测定不同时间的含沙量的垂线分布,运用图积分的方法,即可求出不同 $z$ 处的瞬时沉降速率 $\bar{\omega}$ 随 时间的变化. McLaughlin 命名此法为 “重复深度吸管法” ${ }^{[15]}$, 实质上是 “吸管法” ${ }^{[16]}$ 的进一步改进.

本文根据不同时刻沉降筒上、中、下处 TSM 浓度的值,利用重复深度吸管法,计算太湖悬浮物的沉降速 度. 同时采用水深加权的方法计算每取样时刻沉降筒内的平均浓度,采用浓度加权的方法求取 3 个取样口 的平均沉降速率. 其计算方法如下:

$$
\begin{aligned}
& \bar{C}=\left(h_{1} C_{1}+h_{2} C_{2}+h_{3} C_{3}\right) /\left(h_{1}+h_{2}+h_{3}\right) \\
& \bar{\omega}=\left(\omega_{1} C_{1}+\omega_{2} C_{2}+\omega_{3} C_{3}\right) /\left(C_{1}+C_{2}+C_{3}\right)
\end{aligned}
$$

式(4) 中, $\bar{C}$ 为沉降筒内水深加权平均浓度 $(\mathrm{mg} / \mathrm{L}) ; C_{1}, C_{2}, C_{3}$, 分别为沉降筒上、中、下取样口处 $T S M$ 的浓度 $(\mathrm{mg} / \mathrm{L}) ; h_{1}, h_{2}, h_{3}$ 分别为沉降筒上、中、下取样口离沉降筒上端筒口的距离 $(\mathrm{cm})$. 式 $(5)$ 中, $\bar{\omega}$ 为沉降筒内 浓度加权平均沉降速率 $(\mathrm{cm} / \mathrm{s}) ; \omega_{1}, \omega_{2}, \omega_{3}$ 分别为沉降筒上、中、下取样口高度处平均沉降速率 $(\mathrm{cm} / \mathrm{s})$.

\begin{tabular}{|c|c|c|c|c|c|c|c|c|c|c|}
\hline 实验次数 & $\begin{array}{c}\mathrm{TSM} \text { 初始 } \\
\text { 浓度 }(\mathrm{mg} / \mathrm{L})\end{array}$ & $\begin{array}{c}\text { POM 初始 } \\
\text { 浓度 }(\mathrm{mg} / \mathrm{L})\end{array}$ & $\begin{array}{c}\mathrm{d}(0.5) \text { 中值 } \\
\text { 粒径 }(\mu \mathrm{m})\end{array}$ & $\begin{array}{l}0.02-4 \\
\mu \mathrm{m}(\%)\end{array}$ & $\begin{array}{c}4-8 \\
\mu \mathrm{m}(\%)\end{array}$ & $\begin{array}{c}8-16 \\
\mu m(\%)\end{array}$ & $\begin{array}{c}16-32 \\
\mu \mathrm{m}(\%)\end{array}$ & $\begin{array}{c}32-64 \\
\mu \mathrm{m}(\%)\end{array}$ & $\begin{array}{l}64-125 \\
\mu \mathrm{m}(\%)\end{array}$ & $\begin{array}{c}125-2000 \\
\mu \mathrm{m}(\%)\end{array}$ \\
\hline 第 1 次 & 293.8 & 55.3 & 8.9 & 22.1 & 23.2 & 28.7 & 17.3 & 5.3 & 1.9 & 1.2 \\
\hline 第 2 次 & 48.2 & 32.6 & 7.2 & 28.3 & 25.6 & 24.7 & 11.6 & 3.8 & 2.1 & 3.5 \\
\hline 第 3 次 & 589.5 & 61.1 & 10.0 & 20.6 & 20.3 & 27.4 & 19.9 & 7.4 & 2.1 & 2 \\
\hline 第 4 次 & 52.0 & 19.5 & 7.2 & 29.1 & 24.6 & 27.3 & 15.1 & 2.9 & 0.7 & 0 \\
\hline
\end{tabular}

\section{2 结果与讨论}

\section{1 水体中悬浮物的初始浓度及粒度}

表 1 静沉降实验中悬浮物的初始浓度和粒径

Tab. 1 Initial concentration and particle size of suspended matter in hydrostatic settling experiments

4 次沉降实验中,4 月初进行的 2 次实验, 初始 $T S M$ 浓度分别为 $293.8 \mathrm{mg} / \mathrm{L}$ 和 $48.2 \mathrm{mg} / \mathrm{L}$, 取样时风速 分别为 $7.2 \mathrm{~m} / \mathrm{s}$ 和 $3.2 \mathrm{~m} / \mathrm{s}$, 代表了太湖在春季中大风和小风情况下湖体悬浮物浓度的分布情况; 5 月底进 行的 2 次实验, 初始 $T S M$ 浓度分别为 $589.5 \mathrm{mg} / \mathrm{L}$ 和 $52.0 \mathrm{mg} / \mathrm{L}$, 代表了太湖在初夏大风和小风情况下湖体 悬浮物浓度的分布情况. 表 1 显示, 太湖水体中悬浮物颗粒的粒径主要分布在 0-32 $\mu \mathrm{m}$ 之间, 大于 $125 \mu \mathrm{m}$ 的颗粒所占的比例不到 $4 \%$, 大于 $32 \mu \mathrm{m}$ 的颗粒也不到 $15 \%$. 其它粒径颗粒的分布范围一般均在 $10 \%-$ $30 \%$ 之间.

\section{2 悬浮物浓度与沉降时间的关系}

4 次实验中,水体中悬浮颗粒物的浓度均呈现出随时间呈指数衰减的相似规律. 以第 1 次实验为例, 沉 降筒上、中、下 3 个取样口 TSM 浓度与沉降时间的关系表明 (图 1), 各个取样口处 TSM 浓度在沉降初期下 
降幅度最大, 以后下降幅度逐渐变缓. 在前 $180 \mathrm{~min}$ 内, TSM 浓度能下降到一半以上, 以后的时间中,下降的 幅度逐渐减小, 几个小时甚至十几个小时内 TSM 下降的幅度也只在 $20 \%-40 \%$ 左右. 在垂向上, 在同一沉 降时刻,基本上是下层的 TSM 浓度 > 中层的 TSM 浓度 >上层的 TSM 浓度,形成了垂向的浓度梯度. 在沉降 初期 3 个取样口浓度的差别比沉降后期要大, 反映出了 TSM 在沉降过程中的浓度分布规律.

表 2 不同起始浓度条件下 TSM 浓度与沉降时间的关系 ${ }^{1)}$

Tab. 2 Relationship between TSM and settling time on different initial concentrations

\begin{tabular}{|c|c|c|}
\hline 实验次数 & 取样位置 & $T S M$ 浓度拟合曲线及相关系数 \\
\hline \multirow{3}{*}{ 第 1 次 } & 上层 & $y=26.2+180.0 \mathrm{e}^{-x / 41.8}+90.0 \mathrm{e}^{-x / 817.7}, R^{2}=0.99$ \\
\hline & 中层 & $y=22.5+101.4 \mathrm{e}^{-x / 762.6}+178.0 \mathrm{e}^{-x / 63.3}, R^{2}=0.99$ \\
\hline & 下层 & $y=16.0+119.1 \mathrm{e}^{-x / 869.3}+165.8 \mathrm{e}^{-x / 79.1}, R^{2}=0.99$ \\
\hline \multirow{3}{*}{ 第 2 次 } & 上层 & $v=-11320.7+11362.5 \mathrm{e}^{-x / 1.0 \mathrm{e} 6}+10.7 \mathrm{e}^{-x / 105.2}, R^{2}=0.80$ \\
\hline & 中层 & $v=28.0+105 \mathrm{e}^{-x / 38.0}+19.7 \mathrm{e}^{-x / 618.7}, R^{2}=0.85$ \\
\hline & 下层 & $v=-39.5+44.6 \mathrm{e}^{-x / 4411.7}+44.6 \mathrm{e}^{-x / 4412.0}, R^{2}=0.92$ \\
\hline \multirow{3}{*}{ 第 3 次 } & 上层 & $v=29.3+430.1 \mathrm{e}^{-x / 13.6}+132.6 \mathrm{e}^{-x / 444.8}, R^{2}=0.99$ \\
\hline & 中层 & $v=32.4+141.5 \mathrm{e}^{-x / 395.9}+423.4 \mathrm{e}^{-x / 16.6}, R^{2}=1.00$ \\
\hline & 下层 & $v=20.3+445.9 \mathrm{e}^{-x / 22.2}+126.3 \mathrm{e}^{-x / 754.0}, R^{2}=0.99$ \\
\hline \multirow{3}{*}{ 第 4 次 } & 上层 & $v=6.7+43.2 \mathrm{e}^{-x / 1084.8}, R^{2}=0.86$ \\
\hline & 中层 & $v=4.6+46.2 \mathrm{e}^{-x / 1474.7}, R^{2}=0.82$ \\
\hline & 下层 & $v=7.7+43.1 \mathrm{e}^{-x / 1120.3}, R^{2}=0.91$ \\
\hline
\end{tabular}

$1)$ : 表中 $x$ 为沉降时间 $(\min ), y$ 为 $T S M$ 浓度 $(\mathrm{mg} / \mathrm{L})$; 各次拟合样本容量均为 $n=18$.

各取样口 TSM 浓度与沉降时间关系的拟合结果表明,水 体中 TSM 浓度均随沉降时间呈指数衰减, 尤其是当水体中 $T S M$ 的起始浓度较大时, 这种规律更加明显 $\left(R^{2} \geqslant 0.99\right)($ 表 2 ). 其原因可能主要与水体中悬浮物的组成有关. 实验中, 当 TSM 初始浓度较大时 (第 $1 、 3$ 次实验), 其有机物的含量均 低于 $20 \%$; 而当 TSM 初始浓度较小时 (第 2、4 次实验), 其有 机物含量则分别达到 $67.6 \%$ 和 $37.5 \%$ (表 1). 毫无疑问, 水 体悬浮物中有机质的含量会显著影响其中细颗粒泥沙的絮凝 沉降特性 ${ }^{[17]}$, 当悬浮物中有机物的含量较高时, 其与水体中 其它颗粒相吸附、絮凝的概率也越大, 由于悬浮物的絮凝沉降 速率要比单颗粒悬浮物的沉降速率大得多, 从而导致其沉降 速率、衰减规律也不尽相同.

\section{3 水体中悬浮物的沉降速率}

\subsection{1 斯托克斯公式计算的悬浮物沉降速率四次实验的中}

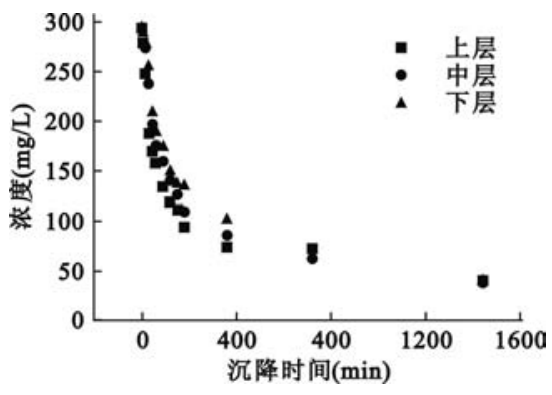

图 1 第 1 次沉降实验中 3 个取样口 $T S M$ 浓度与沉降时间的关系

Fig. 1 Relationship between TSM and settling time of 3 sampling sites in 1 st experiment

值粒径分别为 $0.089 \mathrm{~mm} 、 0.072 \mathrm{~mm} 、 0.010 \mathrm{~mm}$ 和 $0.072 \mathrm{~mm}$, 按斯托克斯公式计算的中值沉速分别为 0.0056 $\mathrm{cm} / \mathrm{s}, 0.0036 \mathrm{~cm} / \mathrm{s}, 0.0070 \mathrm{~cm} / \mathrm{s}$. 由于水体中悬浮物的粒径不同时, 其沉降速度显著不同, 因此不能简单的 仅采用中值粒径来计算太湖悬浮物的沉降速度. 按照式 (1), 在假定有效容重系数 $\alpha=\left(\gamma_{\mathrm{s}}-\gamma\right) / \gamma$ 的值为 $1.65^{[18]}$ 的情况下,用斯托克斯公式计算了不同粒径情况下的沉降速率 (表 3 ). 结果表明,水体中不同粒径 的悬浮物, 其沉降速率差异极大. 当悬浮物的粒径为 $0.001-0.032 \mathrm{~mm}$ 时, 其对应的沉降速率为 $0.0001-$ $0.0719 \mathrm{~cm} / \mathrm{s}$, 而当粒径增至 $0.032-0.125 \mathrm{~mm}$ 时, 其对应的沉降速率急速增大至 $0.0719-1.0964 \mathrm{~cm} / \mathrm{s}$. 如 果以太湖平均水深 $2.0 \mathrm{~m}$ 计, 则呈完全分散或单颗粒状态的悬浮物按静沉降速率计算, 从水面沉至湖底的 时间约为 $0.77-793.7 \mathrm{~h}$ (悬浮物粒径为 $0.001-0.032 \mathrm{~mm}$ ), 而当悬浮物粒径增至 $0.032-0.125 \mathrm{~mm}$ 时, 从 水面沉至湖底仅需 $0.05-0.77 \mathrm{~h}$ (表 3 ). 
表 3 悬浮物粒径与沉降速率的关系 ${ }^{1)}$

Tab. 3 Relationship between particle size and settling velocity of TSM

\begin{tabular}{ccccccccc}
\hline 粒径 $(\mathrm{mm})$ & 0.001 & 0.002 & 0.003 & 0.004 & 0.005 & 0.006 & 0.007 & 0.008 \\
\hline 沉降速率 $(\mathrm{cm} / \mathrm{s})$ & 0.0001 & 0.0003 & 0.0006 & 0.0011 & 0.0018 & 0.0025 & 0.0034 & 0.0045 \\
从沉降筒顶沉至筒底时间 $(\mathrm{min})$ & 11905 & 2976 & 1323 & 744 & 476 & 329 & 242 & 186 \\
从水面沉至湖底时间 $(\mathrm{h})$ & 793.7 & 198.4 & 88.2 & 49.6 & 31.8 & 22.0 & 16.2 & 12.4 \\
\hline 粒径 $(\mathrm{mm})$ & 0.01 & 0.016 & 0.025 & 0.032 & 0.05 & 0.064 & 0.1 & 0.125 \\
\hline 沉降速率 $(\mathrm{cm} / \mathrm{s})$ & 0.0070 & 0.0180 & 0.0439 & 0.0719 & 0.1754 & 0.2874 & 0.7017 & 1.0964 \\
从沉降筒顶沉至筒底时间 $(\mathrm{min})$ & 118.7 & 46.4 & 19.1 & 11.6 & 4.8 & 2.9 & 1.2 & 0.8 \\
从水面沉至湖底时间 $(\mathrm{h})$ & 7.91 & 3.09 & 1.27 & 0.77 & 0.32 & 0.19 & 0.08 & 0.05 \\
\hline
\end{tabular}

1) 在计算沉降速率时,有效容重系数 $\alpha=1.65$.

2.3 .2 重复深度吸管法计算的悬浮物沉降速率 用重复深度吸管法计算的悬浮物沉降速率( 以第 1 次实验 为例)结果见表 4. 综合四次实验的计算结果, 将平均浓度和平均沉降速率进行非线性拟合发现 Logistic 函 数拟合关系最好 (图 2), 所拟合曲线为:

$$
\omega=0.021 /(1+\exp (-0.026 *(C-166.3))),\left(R^{2}=0.98, N=54\right)
$$

式 (6) 中, $\omega$ 为平均沉降速率 $(\mathrm{cm} / \mathrm{s}), C$ 为悬浮物浓度 $(\mathrm{mg} / \mathrm{L})$. 图 2 表明, 当悬浮物浓较低时 $(C<100 \mathrm{mg} /$ $\mathrm{L}$ ), 太湖悬浮物的沉降速率与水体中的悬浮物浓度无明显的相关关系; 而悬浮物浓度较高时 ( C > $100 \mathrm{mg}$ / L), 沉降速率随悬浮物浓度升高而增大. 此外, 随着沉降时间的增加, 悬浮物的平均沉降速率逐渐减小, 当 沉降时间足够长时, 沉降速度趋于稳定. 具体表现为, 在开始沉降的 $180 \mathrm{~min}$ 内, 平均沉降速率呈指数下降, $180 \mathrm{~min}$ 时的平均沉降速率只有初始平均沉降速率的 $1 / 5-1 / 2$. TSM 浓度在前 $180 \mathrm{~min}$ 内变化也较大, 在沉 降初期 TSM 浓度的减少量达 50\% 以上. 在随后 3-6 h 内,沉降速率进一步下降,下降幅度有所减小, 而在 沉降经历了十几小时, 几十小时后沉降速度趋于稳定, 与初始浓度的关系不再是很密切. 各种 TSM 初始浓 度在沉降 $24 \mathrm{~h}$ 后, 沉降速度大都位于 $0.0002-0.0009 \mathrm{~cm} / \mathrm{s}$ 之间, 相差不大. 这说明太湖悬浮物的沉降过 程是一个渐变的过程, 大风浪过后, 在短时间内大颗粒的无机物和部分有机物能够很快沉降下来, 但水体中 的小颗粒有机物和生物碎屑等的完全沉降则需要较长时间.

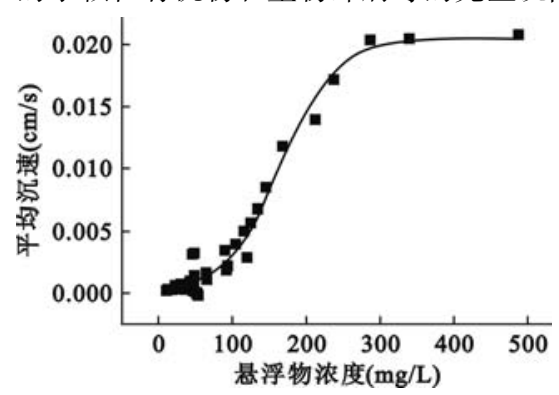

图 2 平均沉降速率 - 悬浮物浓度的关系

Fig. 2 Relationship between TSM concen-

tration and its average settling velocity
2.3 .3 两种计算方法比较与分析 4 次实验中, 悬浮物的中值 粒径均在 7-11 $\mu \mathrm{m}$ 之间 (表 1). 在悬浮物粒径 $<4 \mu \mathrm{m} 、 4-8$ $\mu \mathrm{m} 、 8-16 \mu \mathrm{m} 、 16-32 \mu \mathrm{m}$ 几个范围内, 各粒径范围所占的百分 比较为均匀,均在 $10 \%-30 \%$ 之间. 大于 $32 \mu \mathrm{m}$ 的粒径均在 $3 \%-15 \%$ 之间. 由于悬浮物在下沉过程中,一般为大颗粒的 悬浮物首先下沉, 且沉降速率快, 小颗粒的物质下沉慢. 所以 各个时间段,下沉的主导颗粒粒径不一样, 主导沉降速率也是 不一样的,应该是前期沉降速率大, 后期沉降速率小.

根据斯托克斯公式计算结果, 悬浮物粒径在 50-125 $\mu \mathrm{m}$ 之间的颗粒物沉降速率在 $0.1754-1.0964 \mathrm{~cm} / \mathrm{s}$ 之间. 若沉降 筒中水面到筒底高度以 $54 \mathrm{~cm}$ 计,粒径在 $50-125 \mu \mathrm{m}$ 范围内的 颗粒在 $5 \mathrm{~min}$ 之内能够全部沉入筒底. 依次类推, 粒径大于 32 $\mu \mathrm{m}$ 的颗粒在 $12 \mathrm{~min}$ 内能够全部沉人筒底. 粒径 $<4 \mu \mathrm{m} 、 4-8 \mu \mathrm{m} 、 8-16 \mu \mathrm{m} 、 16-32 \mu \mathrm{m}$ 全部沉人筒底时 间分别为 $12.4-198 \mathrm{~h}, 3-12.4 \mathrm{~h}, 46.4 \mathrm{~min}-3 \mathrm{~h}, 11.6 \mathrm{~min}-46.4 \mathrm{~min}$. 按照此推论, 沉降 $12 \mathrm{~min}$ 后的沉降速 率应小于 $0.0750 \mathrm{~cm} / \mathrm{s}, 46 \mathrm{~min}$ 后的沉降速率应小于 $0.0196 \mathrm{~cm} / \mathrm{s}, 120 \mathrm{~min}$ 后的沉降速率应小于 $0.0075 \mathrm{~cm} /$ $\mathrm{s}, 360 \mathrm{~min}$ 后的沉降速率应小于 $0.0025 \mathrm{~cm} / \mathrm{s}, 720 \mathrm{~min}$ 后的沉降速率应小于 $0.0013 \mathrm{~cm} / \mathrm{s}, 2160 \mathrm{~min}$ 后的沉降 速率应小于 $0.0004 \mathrm{~cm} / \mathrm{s}$, 依次类推. 将其与方法 2 “重复深度吸管法” 的计算结果进行比较可得, 当粒径在 $0-32 \mu \mathrm{m}$ 之间时,方法 2 的绝大多数计算结果刚好落人方法 1 设定的范围内, 对于初始浓度较大的第 3 次 实验, 沉降速率的计算结果大多数大于方法 1 设定的下限值. 由于方法 1 给出的是按沉降筒的最大高度给 
出的沉降速率下限值, 实际上这一值比按平均高度计算的值大很多. 即使这样, 方法 2 沉降速率计算值与 方法 1 沉降速率计算值的下限接近,有的甚至大于方法 1 的计算值. 说明方法 1 的计算值偏小,应采用方法 2 的计算结果. 同时也说明太湖悬浮物的沉降不是单颗粒沉降的,而是絮凝沉降的.

\section{表 4 重复深度吸管法计算的平均沉降速率}

Tab. 4 Average settling velocity of TSM calculated by Mclaughlin method

\begin{tabular}{cccccc}
\hline $\begin{array}{c}\text { 沉降时间 } \\
(\mathrm{min})\end{array}$ & $\begin{array}{c}\text { 水深加权平均浓度 } \\
(\mathrm{mg} / \mathrm{L})\end{array}$ & $\begin{array}{c}\text { 上层取样口处 } \\
\text { 沉降速率 }(\mathrm{cm} / \mathrm{s})\end{array}$ & $\begin{array}{c}\text { 中层取样口处 } \\
\text { 沉降速率 }(\mathrm{cm} / \mathrm{s})\end{array}$ & $\begin{array}{c}\text { 下层取样口处 } \\
\text { 沉速率 }(\mathrm{cm} / \mathrm{s})\end{array}$ 沉度加权速率 $(\mathrm{cm} / \mathrm{s})$ \\
\hline 5 & 287.82 & 0.00241 & 0.00148 & 0.00286 & 0.00225 \\
15 & 272.11 & 0.00267 & 0.00269 & 0.00307 & 0.00282 \\
30 & 239.17 & 0.00351 & 0.00403 & 0.00393 & 0.00385 \\
45 & 198.86 & 0.00286 & 0.00506 & 0.00634 & 0.00487 \\
60 & 193.54 & 0.00242 & 0.00486 & 0.00429 & 0.00393 \\
90 & 162.95 & 0.00204 & 0.00385 & 0.00487 & 0.00371 \\
120 & 141.73 & 0.00176 & 0.00349 & 0.00471 & 0.00343 \\
150 & 129.41 & 0.00152 & 0.00319 & 0.00425 & 0.00308 \\
180 & 119.97 & 0.00148 & 0.00312 & 0.00361 & 0.00286 \\
360 & 91.94 & 0.00090 & 0.00194 & 0.00247 & 0.00185 \\
720 & 65.16 & 0.00045 & 0.00122 & 0.00175 & 0.00111 \\
1440 & 38.84 & 0.00033 & 0.00081 & 0.00116 & 0.00076 \\
\hline
\end{tabular}

此外,对于沉降筒上中下 3 个取样口处的 TSM 浓度,在沉降初期下降的非常快 (图 2). 对于初始浓度 大的第 3 次实验, $20 \mathrm{~min}$ 内 $T S M$ 浓度下降了一半以上,而其悬浮物的中值粒径也只有 $10 \mu \mathrm{m}$,按照方法 1 计 算, 悬浮物全部沉降下来需要 $120 \mathrm{~min}$, 即使只有一半沉降下来也需要 $60 \mathrm{~min}$, 大于实际实验中所需的时间. 说明太湖悬浮物的沉降是絮凝沉降, 方法 1 不适合用来精确计算太湖悬浮物的沉降速率, 但可以进行粗略 估算, 没有量级的差别. 而方法 2 能较好的反映太湖悬浮物的沉降情况,但由于方法 2 在不同时间,不同高 度有不同的沉降速率计算值, 所以在使用方法 2 时要注意根据悬浮物的实际特性, 选取其特征沉降速率.

野外的原位沉降过程还受到风浪扰动、再悬浮、水华以及一些外来力引起的波浪运动等影响, 其沉降特 性较之静沉降复杂得多. 根据表 4, 方法 2 计算的沉降速率集中在 $0.002-0.005 \mathrm{~cm} / \mathrm{s}$ 的范围内. 已有的研 究结果显示, Rosa 测定 Ontario 湖悬浮物的原位沉降速率为 $0.00009 \mathrm{~cm} / \mathrm{s}^{[18]}$, Eadie 等测定 Zug 湖悬浮物沉 降速率为 $0.0022 \mathrm{~cm} / \mathrm{s}^{[19]}$, 有关珠江口的研究表明其淡水区域悬浮物沉降速率为 $0.032 \mathrm{~cm} / \mathrm{s}^{[20]}$, 大多与我 们对太湖的估算值比较接近. 要更为准确地获取太湖悬浮物的沉降速率, 仍有待于后续原位实验的展开和 进一步验证.

\section{3 结论}

(1) 太湖悬浮物在静沉降时,水体中悬浮物浓度与沉降时间呈指数衰减的规律,且悬浮颗粒中有机物 含量越低,悬浮物浓度随时间的变化越接近指数衰减规律.

(2) 利用斯托克斯公式和重复深度吸管法计算了不同粒度太湖悬浮物的沉降速度. 利用斯托克斯公式 计算的太湖悬浮物的沉降速率偏小, 说明太湖悬浮物沉降不属于单颗粒沉降, 而是属于絮凝沉降. 斯托克 斯公式不能用来精确计算太湖悬浮物沉降速率, 但可以用来估算. 而重复深度吸管法适合于精确计算太湖 悬浮物的沉降速率, 太湖悬浮物的沉降速度与水体中的悬浮物浓度有关, 悬浮物浓度越大, 其沉降速率越 大, 在 20-30 min 以内, 悬浮物沉降率可达 50\% 以上. 另外, 随着沉降时间的增加, 悬浮物的平均沉降速率 逐渐减小, 当沉降时间足够长时, 沉降速度趋于稳定. 由于重复深度吸管法在不同水深、不同沉降时间有不 同的沉降速率计算值, 所以在使用该法时要注意根据悬浮物的实际特性,选取其特征沉降速率. 按本文的 方法, 太湖悬浮物的沉降速率范围为 $0.002-0.005 \mathrm{~cm} / \mathrm{s}$. 
(3) 当悬浮物浓较低时,太湖悬浮物的沉降速率与水体中的悬浮物浓度无明显的相关关系;而悬浮物 浓度较高时, 沉降速率随悬浮物浓度升高而增大. 通过对 4 次太湖悬浮物沉降实验结果的统计分析, 发现 悬浮物浓度和平均沉降速率的关系符合 Logistic 曲线, 拟合关系为:

$$
\omega=0.021 /(1+\exp (-0.026 \times(C-166.3))), R^{2}=0.98, N=54 .
$$

\section{4 参考文献}

[1] Qin Boqiang. Dynamics of sediment resuspension and the conceptual schema of nutrient release in the large shallow lake Taihu, China. Chinese Science Bulletin, 2004, 49(1) : 54 -64.

[2] 张运林, 秦伯强, 陈伟民等. 太湖水体中悬浮物研究. 长江流域资源与环境, 2004, 13(3): 266 -271 .

[3] Simon M, Grossart H P, Schweitzer B, et al. Microbial ecology of organic aggregates in aquatic ecosystems. Aquat Microb Ecol, 2002, 28: 175 - 211.

[4] Turner J T. Zooplankton fecal pellets, marine snow and sinking phytoplankton blooms. Aquat Microb Ecol, 2002, 27: $57-102$.

[5] Wang F, Chen J \& Forslin W. Modelling sorption of trace metals on natural sediments by surface complexation model. Environmental Science and Technology, 1997, 31: 448 - 453.

[6] Jannasch H W, Honeyman B D \& Murray J W. Marine scavenging: the relative importance of mass transfer and reaction rates. Limnol Oceanogr, 1996, 41: 82 - 88.

[7] Richard W, Douglas, Brian Rippey, et al. Gibson. Estimation of the in-situ settling velocity of particles in lakes using a time series sediment trap. Freshwater Biology, 2003, 48: 512 - 516.

[8] 雷 坤, 杨作升, 郭志刚. 东海陆架北部泥质区悬浮体的絮凝沉积作用. 海洋与湖沼, 2001, 32(3): $288-295$.

[9] Riley G A, Stommel H \& Bumpus D F. Quantitative ecology of the plankton of the western North Atlantic. Bull Bingham Oceanogr Collec, 1949, 12 : 1 - 169.

[10] Burns N M \& Rosa F. In situ measurement of the settling velocity of organic carbon particles and 10 species of phytoplankton. Limnol Oceanogr, 1980, 25(5) : 855 - 864.

[11] Isabelle Larocque, Mazumder A, Proulx M, Lean D R S, et al. Sedimentation of algae: relationships with biomass and size distribution. Can J Fish Aquat Sci, 1996, 53: 1133 - 1142.

[12] Marc Schallenberg, Carolyn W Burns. Effects of sediment resuspension on phytoplankton production: teasing apart the influences of light, nutrients and algal entrainment. Freshwater Biology, 2004, 49: 143 - 159.

[13] Becquevort S, Smith W O Jr. Aggregation, sedimentation and biodegradability of phytoplankton-derived material during spring in the Ross Sea, Antarctica. Deep-Sea Research II , 2001, 48: 4155 - 4178.

[14] McLaughlin R T Jr. Settling properties of suspension. Trans Am Soc Civ Eng, 126: 1734 - 1767.

[15] 黄建维. 粘性泥沙在静水中沉降特性研究. 泥沙研究, 1981, (2) : $31-49$.

[16] 中国科学院南京土壤研究所. 土壤理化分析. 上海: 上海科学技术出版社, 1978: 474-490.

[17] 陈洪松, 邵明安. 有机质对细颗粒泥沙静水絮凝沉降特性的影响. 泥沙研究, 2001, (3): 35 - 39.

[18] 罗潋苾. 太湖水动力学及其环境效应研究. 中国科学院研究生院博士学位论文,2004.

[18] Rosa F. Sedimentation and sediment resuspension in Lake Ontario. Journal of Great Lakes Research, 1985, 11: $13-25$.

[19] Eadie B J, Vanderploeg H A, Robbins J A, et al. Significance of sediment resuspension and particle settling. In:Tilzer M M \& Serruya C , ed. Large Lakes. New York: Springer-Verlag, 1990: 196 - 209.

[20] Xia X M, Li Y, Yang H et al. Observations on the size and settling velocity distributions of suspended sediment in the Pearl River Estuary, China. Continental Shelf Research, 2004, 24: 18 - 26. 